\section{Evaluation of the}

\section{pharmacokinetics of digoxin} in healthy subjects receiving etoricoxib

\section{Correspondence}

Dr Jules I. Schwartz, Merck Research Laboratories, 126 E. Lincoln Avenue, PO Box 2000, RY34-A552, Rahway, NJ 07065-0900, USA

Tel: +17325945838

Fax: + 17325945405

E-mail:jules_schwartz@merck.com

\section{Keywords}

digoxin, etoricoxib, pharmacokinetics

\section{Received}

Jules I. Schwartz, Nancy G. B. Agrawal, ${ }^{1}$ Martin Wehling, ${ }^{2}$

Bret J. Musser, Carol P. Gumbs, Nicole Michiels, Marina De Smet \& John A. Wagner

Merck Research Laboratories, Rahway, NJ and ${ }^{~}$ Merck \& Co., Inc., West Point, PA, USA, and ${ }^{2}$ University of Heidelberg, Heidelberg, Germany

\section{WHAT IS ALREADY KNOWN ABOUT} THIS SUBJECT

- Digoxin is a well-recognized inotropic agent

with a narrow therapeutic index.

- Nonsteroidal anti-inflammatory drugs, including the selective cyclooxygenase-2 inhibitor etoricoxib, are very likely to be used in patients receiving digoxin due to the similar populations requiring the drugs.

- Digoxin drug interactions should be assessed and described to clinicians when new drugs become available.

\section{WHAT THIS STUDY ADDS}

- This study is the first to provide the practitioner with a detailed description of the influence of etoricoxib on the pharmacokinetics of digoxin.

- Due to the methods used, it shows that etoricoxib does not interfere with P-glycoprotein in the kidney, a method of digoxin disposition.

- In addition, this paper provides confidence to the practitioner that there will be no clinically important reason to avoid the combination of the two drugs.

\section{AIMS}

Digoxin is a commonly prescribed cardiac glycoside with a narrow therapeutic index. The aim was to investigate whether the cyclooxygenase- 2 selective nonsteroidal anti-inflammatory drug etoricoxib affects the steady-state pharmacokinetics of digoxin.

\section{METHODS}

This was a double-blind, randomized, placebo-controlled, two-period cross-over study. In each period, 14 healthy volunteers ranging in age from 21 to 35 years received oral digoxin $0.25 \mathrm{mg}$ daily and were randomized to either etoricoxib $120 \mathrm{mg}$ or matching placebo tablets once daily for 10 days. Trough digoxin plasma concentrations were analysed by linear regression to examine digoxin accumulation over time.

The geometric mean ratios (etoricoxib/placebo) for $\mathrm{AUC}_{0-24 \mathrm{~h}}, \mathrm{C}_{\max }$ and urinary excretion were 1.06 (90\% confidence interval $0.97,1.17), 1.33$ $(1.21,1.46)$ and $1.10(1.00,1.20)$, respectively. The median (range) for digoxin $T_{\max }(\mathrm{h})$ values with etoricoxib and placebo were $0.5(0.5,1.5)$ and $1.0(0.5,1.5)$, respectively. Steady-state digoxin plasma concentrations were achieved by day 7 in each treatment period. No serious adverse experiences were reported.

\section{CONCLUSIONS}

Although etoricoxib $120 \mathrm{mg}$ did produce an approximately $33 \%$ increase in digoxin $C_{\max }$, this increase does not appear to be clinically meaningful, as cardiotoxicity with digoxin has been associated with elevations in steady-state rather than peak concentrations. From these results, it appears that etoricoxib does not cause any changes in digoxin steady-state pharmacokinetics that would necessitate a dose adjustment.

\section{RESULTS}




\section{Introduction}

Nonsteroidal anti-inflammatory drugs (NSAIDs) are highly effective for the treatment of pain and inflammation and are one of the most widely prescribed classes of drug worldwide. It has been estimated that as many as $70 \%$ of people $>65$ years old take at least one dose of an NSAID each week. Approximately one-third of this age group takes at least seven doses of an aspirin or non-aspirin NSAID per week [1]. Cyclooxygenase-2 (COX-2)-selective NSAIDs were developed as a strategy to reduce the risk of gastrointestinal (Gl) clinical events, such as bleeding ulcers, that are associated with the inhibition of COX-1 in the Gl mucosa and COX-1-dependent platelet function by traditional NSAIDs.

Etoricoxib is a COX-2-selective NSAID [2] that is effective for the treatment of acute pain and the pain and inflammation associated with chronic conditions such as osteoarthritis, rheumatoid arthritis, ankylosing spondylitis and lower back pain [3]. Because of its COX-2 selectivity, its long-term use is associated with a lower risk of upper GI clinical events compared with traditional NSAIDs [4-6].

Because of the potential widespread use of this COX2-selective NSAID in patient populations with comorbid conditions who are also taking other therapies, it was important to evaluate potential pharmacokinetic interactions of etoricoxib with commonly prescribed medications having a narrow therapeutic index. Here we report the results of a study specifically designed to examine potential pharmacokinetic interactions of etoricoxib $120 \mathrm{mg}$ with the cardiac glycoside digoxin, commonly prescribed for cardiac arrhythmias, mostly atrial fibrillation, and congestive heart failure, predominantly in the elderly population. Although no significant drug interactions were predicted, digoxin and etoricoxib are likely to be co-administered in this population, who commonly need both analgesic and cardiac medication. The elimination half-life of digoxin is approximately $36 \mathrm{~h}$, and clearance is primarily via passive glomerular filtration and Pglycoprotein (P-gp)-mediated active tubular secretion in the kidney [7]. Traditional NSAIDs and COX-2-selective NSAIDs inhibit synthesis of prostaglandins and have been shown to have similar effects on renal function, as measured by minor dose-dependent changes in urinary prostaglandin secretion, glomerular filtration rate and sodium retention [8-13]. Due to digoxin's low therapeutic index and the potential of etoricoxib to inhibit renal function, and thus affect digoxin clearance, it was necessary to determine whether co-administration of etoricoxib could alter the pharmacokinetics of digoxin.

\section{Methods}

\section{Subjects}

The protocol for this study was approved by the Institutional Review Board for the clinical study centre (Institut für Klinische Pharmakologie, Klinikum Mannheim, Germany). All subjects provided written informed consent prior to participation.

We enrolled 14 subjects, eight men and six women, with a mean age of 21.4 years (Table 1). All subjects were determined to be healthy based on their medical history, physical examination, vital signs, electrocardiogram (ECG) and routine laboratory tests. Subjects were required to be within $30 \%$ of ideal body weight based on a standard height and weight table [14]. Persons with a past history of GI abnormalities except for uncomplicated appendectomy, cholescystectomy or colorectal surgery for polyps, nonmalignant tumours or diverticular obstruction were excluded. Subjects were also excluded if their sitting systolic blood pressure (BP) was $>140 \mathrm{mmHg}$ or diastolic BP $>90 \mathrm{mmHg}$. Women of child-bearing potential enrolled into the study were determined to be in the nongravid state based on serum $\beta$-human chorionic gonadotropin measurements and were instructed to use barrier contraceptives throughout the trial.

Subjects were not allowed to take nonstudy medications for 2 weeks prior to study start until the completion of the trial, except for paracetamol for minor ailments at the discretion of the investigator. Subjects were also instructed to avoid excess alcohol and strenuous physical activity for the duration of the study and follow-up period. Subjects were not allowed to drink beverages containing quinine or $>6$ cups of coffee or equivalent intake of caffeine per day.

\section{Study design}

This was a randomized, double-blind, placebo-controlled, two-period cross-over study (protocol 030). A wash-out of $\geq 14$ days (equivalent to 7-9 half-lives, given the digoxin half-life of 1.5-2 days) between periods minimized the

\section{Table 1}

Baseline demographics of the study subjects

\begin{tabular}{|lllr}
\hline Group & Mean (range) Age (years) & Mean (range) Weight (kg) & Mean (range) Height (cm) \\
\hline Men $(\boldsymbol{n}=\mathbf{8})$ & $26.3(21-35)$ & $75.4(60.0-87.3)$ & $179.5(174.0-190.0)$ \\
\hline Women $(\boldsymbol{n}=\mathbf{6})$ & $26.0(23-29)$ & $65.2(54.0-72.0)$ & $166.0(160.0-172.0)$ \\
Total $(\boldsymbol{n}=\mathbf{1 4})$ & $26.1(21-35)$ & $71.0(54.0-87.3)$ & $173.7(160.0-190.0)$ \\
\hline
\end{tabular}


possibility of any carryover effects. The study was specifically designed to test the primary hypothesis that the $90 \%$ confidence interval $(\mathrm{Cl})$ of the steady-state area under the plasma digoxin concentration-time curve $\left(\mathrm{AUC}_{0-24 \mathrm{~h}}\right)$ ratio [(digoxin + etoricoxib)/(digoxin + placebo)] would be contained in the interval 0.8-1.25.

During each 10-day period, subjects received daily oral doses of digoxin $0.25 \mathrm{mg}$ (Lanoxin ${ }^{\mathrm{TM}}$ tablets) and either etoricoxib $120 \mathrm{mg}$, given as one $60-\mathrm{mg}$ and two $30-\mathrm{mg}$ tablets, or matching placebo tablets once daily. A computerized allocation schedule was used to assign subjects to treatment groups. Subjects were required to fast from food or drink except water for $8 \mathrm{~h}$ preceding the first and last doses. Starting on day 1 , all subjects received their study medication with $240 \mathrm{ml}$ of water between $08.00 \mathrm{~h}$ and $09.00 \mathrm{~h}$ each morning under the supervision of study personnel. Breakfast and lunch were served 2 and $6 \mathrm{~h}$ after dosing, respectively, on days $1-9$. On day 10 , a lunch $4 \mathrm{~h}$ after dosing and dinner $10 \mathrm{~h}$ postdose were served. At the discretion of the investigator, subjects were allowed to leave the clinical research unit after the 6-h postdose ECG was performed on days $1-9$. On day 10 , subjects were required to remain in the clinical research unit until all procedures were completed over the 24-h postdose period.

ECGs and vital signs were monitored prior to and following dosing at predefined intervals on days 1-9. Serum electrolytes (potassium, magnesium, calcium) were measured on days 4, 7 and 9 . Blood samples for plasma digoxin analysis were collected in heparinized glass tubes predose on days $1,4,7,8,9,10$ and 11 and at $0 \mathrm{~h}$ (predose digoxin), $0.5,1,1.5,2,3,4,6,8,12,16$ and $24 \mathrm{~h}$ after the digoxin dose on day 10. Samples were centrifuged and plasma aliquoted into new containers and stored at $-20^{\circ} \mathrm{C}$ until analysed. On day 4 only, plasma digoxin levels were determined as quickly as possible after the blood draw at a local laboratory. This was performed to ensure that plasma trough concentrations of digoxin had not exceeded $3 \mathrm{ng} \mathrm{ml}^{-1}$.

The total urinary recovery of digoxin was determined for each subject. Urine samples were collected for the following intervals: $2 \mathrm{~h}$ predose on day 1 and $0-6,6-12$ and 12-24 $\mathrm{h}$ after the digoxin dose on days 10-11.

Concentrations of digoxin in urine and plasma were determined using a radioimmunoassay as previously described [15].The lower limit of detection of the assay was $0.1 \mathrm{ng} \mathrm{ml}^{-1}$. For measurements of digoxin in plasma, interday variation was assessed by preparing quality control (QC) standards of digoxin in plasma of approximately 0.5 , 0.9 and $2 \mathrm{ng} \mathrm{ml}^{-1}$; these QC standards were divided into aliquots of $0.3 \mathrm{ml}$ and stored frozen at $-20^{\circ} \mathrm{C}$ until analysis. The mean concentrations of digoxin in plasma were determined by analysing replicate $(n=6)$ QC standards, giving $0.50 \pm 0.04 \mathrm{ng} \mathrm{ml}^{-1}$ (mean $\pm \mathrm{SD}$ ), coefficient of variation $(\mathrm{CV})=8.7 \%$ for $0.5 \mathrm{ng} \mathrm{ml}^{-1}, 0.89 \pm 0.06 \mathrm{ng} \mathrm{ml}^{-1}, \mathrm{CV}=6.3 \%$ for $0.9 \mathrm{ng} \mathrm{ml}^{-1}$, and $2.04 \pm 0.08 \mathrm{ng} \mathrm{ml}^{-1}, \mathrm{CV}=3.8 \%$ for $2 \mathrm{ng} \mathrm{ml}^{-1}$. QC standards in plasma were assayed daily
( $n=19$ days) with study samples, giving values of $0.54 \pm$ $0.04 \mathrm{ng} \mathrm{ml}^{-1}, \mathrm{CV}=8.1 \%, 0.82 \pm 0.05 \mathrm{ng} \mathrm{ml}^{-1}, \mathrm{CV}=6.2 \%$ and $2.20 \pm 0.08 \mathrm{ng} \mathrm{ml}^{-1}, \mathrm{CV}=3.6 \%$. For measurement of digoxin in urine, variation was assessed by preparing QC standards of digoxin in urine of approximately 1.6, 10 and $40 \mathrm{ng} \mathrm{ml}^{-1}$; these QC standards were divided into aliquots of $0.3 \mathrm{ml}$ and stored frozen at $-20^{\circ} \mathrm{C}$ until analysis. The controls of 10 and $40 \mathrm{ng} \mathrm{ml}^{-1}$ were diluted 10 -fold. The mean concentration of digoxin in urine was determined by analysing replicate $(n=6)$ QC standards, giving $1.63 \pm 0.10 \mathrm{ng} \mathrm{ml}^{-1}, \mathrm{CV}=6.4 \%$ for $1.6 \mathrm{ng} \mathrm{ml}^{-1}, \quad 10.00 \pm 0.86 \mathrm{ng} \mathrm{ml}^{-1}, \quad \mathrm{CV}=8.6 \%$ for $10 \mathrm{ng} \mathrm{ml}^{-1}$, and $36.68 \pm 1.57 \mathrm{ng} \mathrm{ml}^{-1}, \quad \mathrm{CV}=4.3 \%$ for $40 \mathrm{ng} \mathrm{ml}^{-1}$. QC standards in urine were assayed daily ( $N=6$ days), yielding values of $1.60 \pm 0.08 \mathrm{ng} \mathrm{ml}^{-1}$, $\mathrm{CV}=5.2 \%, 10.00 \pm 0.43 \mathrm{ng} \mathrm{ml}^{-1}, \mathrm{CV}=4.3 \%$, and $37.91 \pm$ $1.45 \mathrm{ng} \mathrm{ml}^{-1}, \mathrm{CV}=3.8 \%$.

\section{Pharmacokinetic parameters}

Actual sampling times relative to the timing for dosing on day 10 were used to estimate pharmacokinetic parameters for immunoreactive digoxin for each treatment: $\mathrm{AUC}_{0-24 \mathrm{~h}}$, maximum concentration of drug observed in the plasma $\left(C_{\max }\right)$, and time to reach maximum concentration $\left(T_{\max }\right)$. The $A \cup C_{0-24 h}$ was calculated using the linear trapezoidal method. The values for $C_{\max }$ and $T_{\max }$ were obtained by inspection of the concentration-time data during actual sampling times. Concentration values in concentrationtime profiles that fell below the assay's limit of quantification $\left(0.1 \mathrm{ng} \mathrm{ml}^{-1}\right)$ were replaced by concentration values of one-half of the limit of quantification $\left(0.05 \mathrm{ng} \mathrm{m}^{-1}\right)$ prior to pharmacokinetic analysis. Urinary concentrations and urine volumes from individual collection intervals were used to calculate the total recovery of immunoreactive digoxin in urine over $24 \mathrm{~h}$.

\section{Sample size and data analysis}

Sample size calculations Sample size calculations relied on a pooled estimate of the within-subject SD of the $\mathrm{AUC}_{0-24 \mathrm{~h}}$ of $0.153\left(\mathrm{In} \mu \mathrm{g} \mathrm{m} \mathrm{m}^{-1}\right)$. This was derived from three previous clinical trials that examined potential drug interactions with digoxin [16-18]. A sample size of 14 subjects provided at least $93.9 \%$ probability of yielding $90 \%$ Cls for the (etoricoxib + digoxin)/(placebo + digoxin) geometric mean ratio (GMR) for $A \cup C_{0-24 h}$ of $0.80-1.25$ if the true GMR was 1.0. There was $80 \%$ power to detect a GMR within $0.94-1.07$ with a $90 \% \mathrm{Cl}$ of $0.80-1.25$.

Analysis of pharmacokinetic parameters Digoxin steadystate pharmacokinetic parameters were analysed using an ANOVA model appropriate for a two-period, crossover design. The ANOVA model included terms for sequence, subject-within-sequence, treatment, and period. A log transformation was applied to the $\mathrm{AUC}_{0-24 \mathrm{~h}}$ data. The normality assumption was tested using the Shapiro-Wilk statistic; the homogeneity of variance assumption was evaluated using graphical methods. Similar methodology 
was applied to $C_{\max }$, which was log transformed, and to $T_{\max }$ which was ranked prior to the ANOVA model.Slight nonconstant variance was apparent in a residual plot for $C_{\max }$. Transforming $C_{\max }$ to $1 /\left(C_{\max }\right)^{2}$ in the ANOVA produced a model that met all assumptions; however, the inference from that model was not substantially different from that using $\ln \left[C_{\max }\right]$, indicating that the initial ANOVA model was robust to the nonconstant variance. Thus, this model was not pursued, and only results from the log transformation of $C_{\max }$ are provided. To answer the primary hypothesis, a $90 \% \mathrm{Cl}$ based on the $t$-distribution was generated from the above ANOVA model for the $A_{U C} C_{0-24 h} G M R$ (digoxin + etoricoxib)/(digoxin + placebo). This interval was compared with the clinically meaningful bounds of $(0.80,1.25)$. A similar interval was constructed for $C_{\max }$.

Total urinary excretion of immunoreactive digoxin after concomitant use of either etoricoxib or placebo was compared using an ANOVA model similar to that used for $A \cup C_{0-24 h}$. The normality assumption was tested using the Shapiro-Wilk statistic; the homogeneity of variance assumption was evaluated using graphical methods. The assumptions were satisfied. The achievement of steady state was assessed by determining the day after which the trough concentrations were approximately constant. For each day on which the trough concentration was measured (days 4 and 7-11), plasma concentrations were modelled as a linear function of time (i.e. using linear regression). This regression model was repeated by dropping each successive day (starting with dropping day 4) until the slope was not significantly different from 0.0 . This analysis was done within each treatment group and used a model that included terms for subject [13 degrees of freedom (d.f.)] and day (1 d.f.).

\section{Results}

All 14 subjects completed the trial. The slope of the relationship between plasma trough concentrations of digoxin (predose) vs. study day on days 7,8,9,10 and 11 for subjects in either treatment group did not differ from 0 $(P>0.200)$, indicating that steady-state digoxin concentrations in plasma were obtained prior to the pharmacokinetic evaluations on day 10 . On average, steady-state plasma concentrations of immunoreactive digoxin were attained by day 7 .

The mean plasma concentration-time profiles for immunoreactive digoxin in the presence and absence of concomitant treatment with etoricoxib $120 \mathrm{mg}$ are displayed in Figure 1. The plasma immunoreactive digoxin geometric mean $\mathrm{AUC}_{0-24 \mathrm{~h}}$ values for digoxin plus etoricoxib and digoxin plus placebo were 13.3 and $12.5 \mathrm{ng} \mathrm{h} \mathrm{m}^{-1}$, respectively. The observed difference in the digoxin $A \cup C_{0-24 h}$ during etoricoxib treatment was not significant $(P=0.246)$. The $\mathrm{GMR}$ for $\mathrm{AUC}_{0-24 \mathrm{~h}}$ was 1.06 with a $90 \% \mathrm{Cl}$ of $(0.97,1.17)$. The $90 \% \mathrm{Cl}$ was within the prespecified clinical

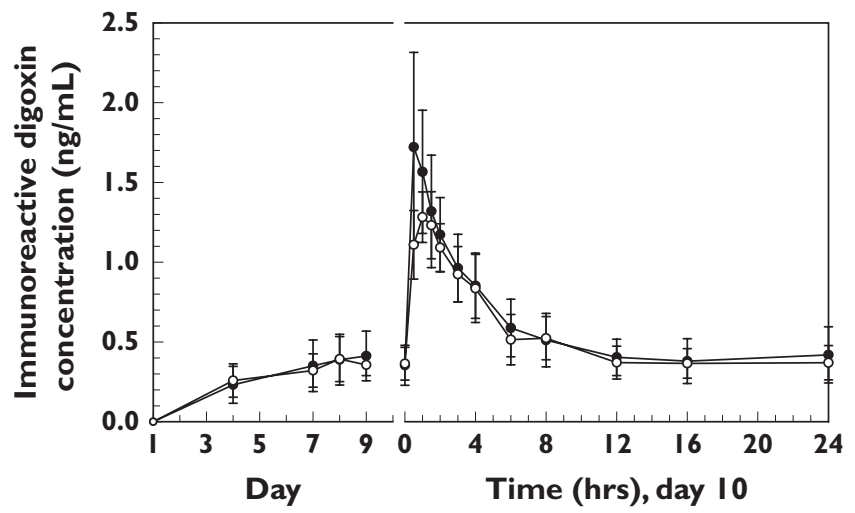

\section{Figure 1}

Mean $( \pm S D)$ concentrations of immunoreactive digoxin in plasma following administration of daily doses of $0.25 \mathrm{mg}$ digoxin and $120 \mathrm{mg}$ etoricoxib or placebo for 10 days. Etoricoxib $(\bullet)$; placebo $(\bigcirc)$

interval of $(0.80,1.25)$, the limits generally accepted for bioequivalence. The highest GMR observed for this parameter was 1.26.

There was a modest but statistically significant $(P<0.001)$ increase in digoxin $C_{\max }$ concentration when administered with etoricoxib $120 \mathrm{mg}$ (Figure 1, Table 2). The GMR for $C_{\max }$ was $1.33(90 \% \mathrm{Cl} 1.21,1.46)$. This increase in digoxin plasma concentration was no longer apparent beyond $4 \mathrm{~h}$ post dose. There was no difference in median $T_{\max }$ values for digoxin when administered with etoricoxib and placebo $(0.5 \mathrm{~h}$ and $1.0 \mathrm{~h}$, respectively; $P=0.087)$.

Total urinary excretion of immunoreactive digoxin on day 10 is summarized in Table 2. There was no difference in geometric mean excretion between the etoricoxib and placebo groups. The GMR for urinary excretion of digoxin was $1.10(90 \% \mathrm{Cl} 1.00,1.21 ; P=0.086)$.

\section{Safety}

All patients received ECGs $6 \mathrm{~h}$ following dosing, and no clinically significant deviations were observed. Nine subjects experienced a total of 15 adverse experiences, nine (five etoricoxib, four placebo) of which were judged by the investigator to be possibly drug-related. These adverse events included headache, bitter taste sensation, and nausea.

\section{Discussion}

Digoxin is frequently used to treat arrhythmias, mostly atrial fibrillation, and heart failure. Because digoxin has a narrow therapeutic index and the possibility exists that both etoricoxib and digoxin may be prescribed to the same patients, this study was conducted to investigate the effect of etoricoxib $120 \mathrm{mg}$ on the pharmacokinetics of digoxin. The 120-mg dose of etoricoxib is the dose recom- 
Table 2

Summary statistics for digoxin $A U C_{0-24 h}, C_{\max }, T_{\max }$ and urinary excretion following a single dose of $0.25 \mathrm{mg}$ oral digoxin in healthy volunteers ( $n=14$ ) receiving daily oral doses of etoricoxib $120 \mathrm{mg}$ or placebo

\begin{tabular}{|c|c|c|c|c|c|}
\hline Parameter Day 10 & Digoxin + Etoricoxib & Digoxin + Placebo & $\mathrm{CV}^{*}$ & GMR Etoricoxib/placebo & $90 \% \mathrm{Cl}$ for GMR \\
\hline$A \cup C_{0-24 h}\left(\mathrm{ng} \mathrm{h} \mathrm{ml}^{-1}\right)$ & $13.3 \pm 4.25 \neq$ & $12.5 \pm 2.38 \neq$ & $13.6 \%$ & 1.06 & $(0.97,1.17)$ \\
\hline$C_{\max }\left(\mathrm{ng} \mathrm{ml}^{-1}\right)$ & $1.76 \pm 0.52 \ddagger$ & $1.32 \pm 0.18 \ddagger$ & $14.4 \%$ & 1.33 & $(1.21,1.46)$ \\
\hline$T_{\max }(\mathrm{h})$ & $0.5(0.5,1.5) \dagger$ & $1.0(0.5,1.5) \dagger$ & & & \\
\hline
\end{tabular}

AUC, Area under the plasma concentration-time curve; $C_{\max }$ maximum concentration of drug observed in the plasma; $T_{\max }$, time to reach maximum concentration; GMR, geometric mean ratio; $\mathrm{Cl}$, confidence interval. *Coefficient of variation (CV) $=100 \times$ root mean square error from the analysis of variance (ANOvA) model (within-subject variation). Ranges were 0.59-1.26 for AUC, 1.00-2.18 for $C_{\max }$. TMedian (min, max). ‡Geometric mean \pm back-transformed SD. The SD was computed as follows: $\operatorname{SQRT}(\operatorname{EXP}(S T D * * 2) *(\operatorname{EXP}(S T D * * 2)-1))^{*}($ Back-Transformed Mean $)$, where STD is the log-scale SD.

mended for the treatment of acute pain and active gouty arthritis. It is greater than the highest daily dose $(90 \mathrm{mg})$ recommended for chronic use [3]. The 0.25 -mg chronic oral dose of digoxin was expected to and did result in readily detectable plasma immunoreactive digoxin concentrations, with no serious adverse experiences. Digoxin was administered together with etoricoxib $120 \mathrm{mg}$ in order to maximize the probability of detecting any drug interaction if patients took the two drugs simultaneously. No interaction was expected, as NSAIDs, including the COX-2selective inhibitor rofecoxib, have not been shown to have clinically important interactions when administered concomitantly with digoxin $[19,20]$.

Examination of the mean concentration-time profiles and the individual pharmacokinetic parameters in the current study revealed that etoricoxib $120 \mathrm{mg}$ has no clinically meaningful effect on immunoreactive digoxin pharmacokinetics. Steady-state AUCs of immunoreactive digoxin were similar between etoricoxib $120 \mathrm{mg}$ and placebo treatments. Additionally, the individual digoxin $A \cup C_{0-24 h}$ GMR were similar, with the exception of one subject who had an $A U C_{0-24 h}$ GMR of 0.59. Excluding data from this subject resulted in a GMR for the remaining subjects combined of $1.12(90 \% \mathrm{Cl} 1.08,1.16)$, which was still within the prespecified $90 \% \mathrm{Cl}$. No explanation is apparent for the decreased digoxin levels observed in this subject since all study drug doses were witnessed. The highest GMR for digoxin $A \cup C_{0-24 h}$ was 1.26. All other subjects had GMRs of $\leq 1.21$ for this pharmacokinetic parameter.

Digoxin is a substrate for P-gp, an energy-dependent efflux pump that regulates the renal tubular secretion of digoxin. P-gp plays a significant role in excreting drugs into urine, so inhibition of the transporting function of P-gp can cause clinically significant drug interactions. Other drugs that inhibit P-gp, including ciclosporin, erythromycin, clarithromycin, propafenone, itraconazole, amiodarone, verapamil and diltiazem, can inhibit digoxin renal elimination and increase digoxin plasma concentration. [21] Whether etoricoxib is a substrate or inhibitor of P-gp has not been tested. Urinary recovery of immunoreactive digoxin in the present study was similar between treatments, however, suggesting that etoricoxib $120 \mathrm{mg}$ does not alter digoxin renal clearance and thus does not meaningfully influence P-gp-mediated transport.

Mean immunoreactive digoxin trough concentrations (days 7-11) were similar between placebo and etoricoxib treatments. Examination of the mean immunoreactive digoxin trough concentrations showed that steady state was achieved by day 7 of multiple dosing when either etoricoxib $120 \mathrm{mg}$ or placebo were administered concurrently with digoxin.

Co-administration of etoricoxib $120 \mathrm{mg}$ did produce a modest increase in digoxin $C_{\max }$ (approximately $33 \%$ ). Although the reason for this increase is unclear, it could potentially be due to a faster rate of drug absorption. This increase in $C_{\max }$ was not seen in a similar study of the effect of rofecoxib, another COX-2-selective NSAID, on digoxin pharmacokinetics, so it does not appear to be a class effect [20]. This increase in plasma digoxin concentration observed during etoricoxib treatment was transient and no longer apparent beyond $4 \mathrm{~h}$ post dose. In fact, nearly identical plasma concentrations for digoxin were observed in the presence and absence of etoricoxib treatment over the $4-24 \mathrm{~h}$ postdose period. In clinical practice, blood samples for therapeutic drug monitoring of digoxin are drawn at least $6 \mathrm{~h}$ after administration of the last dose of digoxin in order to ensure adequate distribution between plasma and myocardial compartments [7, 22, 23]. Hence, samples obtained during distribution $(<6 \mathrm{~h})$ are thought not to be interpretable with regard to prediction of digoxin toxicity $[7,22,23]$. Also, evidence in dogs suggests that the cardiotoxic effect of digoxin is related to elevated steadystate serum concentrations and not to transient increase in peak concentrations, as seen here [24].

In the present study, the digoxin $T_{\max }$ value was $\leq 1.5 \mathrm{~h}$ post dose for all subjects in all treatment periods. Thus, in clinical practice, the modest transient increases in digoxin concentrations observed around $T_{\max }$ during etoricoxib treatment, without any clinically relevant accompanying increase in steady-state AUCs, are unlikely to result in digoxin toxicity or to be of clinical relevance. 
Etoricoxib 120 mg, administered either alone or in combination with digoxin, was well tolerated in this study. In particular, no clinically significant deviations were observed in ECGs obtained $6 \mathrm{~h}$ after dosing, the time of peak pharmacodynamic activity of digoxin [7].

Once-daily co-administration of etoricoxib $120 \mathrm{mg}$ and digoxin $0.25 \mathrm{mg}$ was well tolerated in this small study performed in healthy volunteers. In the recently completed etoricoxib Multinational Etoricoxib and Diclofenac Longterm (MEDAL) clinical trials programme $[25,26]$ enrolling $>34000$ patients with osteoarthritis or rheumatoid arthritis, daily etoricoxib 60 or $90 \mathrm{mg}$ for a mean duration of 18 months was demonstrated to have comparable risk of thrombotic cardiovascular events vs. the traditional NSAID diclofenac $150 \mathrm{mg}$. However, in the MEDAL programme, important increases in renovascular end-points (e.g. fluid retention and blood pressure) were observed with etoricoxib compared with diclofenac. Specifically, the incidence of confirmed congestive heart failure (etoricoxib $90 \mathrm{mg}$ ) and discontinuations due to hypertension (etoricoxib 60 and $90 \mathrm{mg}$ ) were higher with etoricoxib [26]. Due to its effects on the renovascular system, etoricoxib use is contraindicated in patients with New York Heart Association grade II-IV congestive heart failure.

In conclusion, etoricoxib $120 \mathrm{mg}$ co-administered with digoxin $0.25 \mathrm{mg}$ does not produce clinically important effects on the steady-state plasma $A \cup C_{0-24 h}, T_{\max }$ or urinary excretion of immunoreactive digoxin. Although co-administration of etoricoxib $120 \mathrm{mg}$ did produce a modest increase in digoxin $C_{\max }$ (approximately 33\%), this increase was not thought to be clinically meaningful, as plasma digoxin concentrations returned to the levels seen for co-administration with placebo over the 4-24-h postdose period. Based on these pharmacokinetic data in healthy volunteers, no dose adjustment of digoxin appears necessary when co-administered with etoricoxib $120 \mathrm{mg}$ for patients where the use of either agent is not contraindicated.

\section{Competing interests}

J.I.S. N.G.B.A., B.J.M., C.P.G., N.M., M.D.S. and J.A.W. are employees of Merck \& Co., Inc. and may own stock or hold stock options in the company.

This study was supported by Merck Research Laboratories. The authors thank Paul Cavanaugh, Carolyn Hustad and Jennifer Pawlowski of Merck Research Laboratories for assistance with preparation of the manuscript.

\section{REFERENCES}

1 Talley NJ, Evans JM, Fleming KC, Harmsen WS, Zinsmeister AR, Melton LJ III. Nonsteroidal antiinflammatory drugs and dyspepsia in the elderly. Dig Dis Sci 1995; 40: 1345-50.
2 Dallob A, Hawkey CJ, Greenberg H, Wight N, De Schepper P, Waldman S, Wong P, DeTora L, Gertz B, Agrawal N, Wagner J, Gottesdiener K. Characterization of etoricoxib, a novel, selective COX-2 inhibitor. J Clin Pharmacol 2003; 43: 573-85.

3 Matsumoto AK, Cavanaugh PF Jr. Etoricoxib. Drugs Today 2004; 40: 395-414.

4 Laine L, Curtis SP, Cryer B, Kaur A, Cannon CP. Assessment of upper gastrointestinal safety of etoricoxib and diclofenac in patients with osteoarthritis and rheumatoid arthritis in the Multinational Etoricoxib and Diclofenac Long-term (MEDAL) programme: a randomised comparison. Lancet 2007; 369: 465-73.

5 Hunt RH, Harper S, Watson DJ, Yu C, Quan H, Lee M, Evans JK, Oxenius $\mathrm{B}$. The gastrointestinal safety of the COX-2 selective inhibitor etoricoxib assessed by both endoscopy and analysis of upper gastrointestinal events. Am J Gastroenterol 2003; 98: 1725-33.

6 Hunt RH, Harper S, Callegari P, Yu C, Quan H, Evans J, James C, Bowen B, Rashid F. Complementary studies of the gastrointestinal safety of the cyclo-oxygenase-2-selective inhibitor etoricoxib. Aliment Pharmacol Ther 2003; 17: 201-10.

7 Mutnick AH. Digoxin. In: Therapeutic Drug Monitoring, ed Schumacher GE. Norwalk, CT: Appleton \& Lange, 1995; 469-91.

8 Harris RC Jr. Cyclooxygenase-2 inhibition and renal physiology. Am J Cardiol 2002; 89: 10D-17D.

9 Gertz BJ, Krupa D, Bolognese JA, Sperling RS, Reicin A. A comparison of adverse renovascular experiences among osteoarthritis patients treated with rofecoxib and comparator non-selective non-steroidal anti-inflammatory agents. Curr Med Res Opin 2002; 18: 82-91.

10 Curtis SP, Ng J, Yu Q, Shingo S, Bergman G, McCormick CL, Reicin AS. Renal effects of etoricoxib and comparator nonsteroidal anti-inflammatory drugs in controlled clinical trials. Clin Ther 2004; 26: 70-83.

11 Schwartz Jl, Vandormael K, Malice MP, Kalyani TN, Lasseter KC, Holmes GB, Gertz BJ, Gottesdiener KM, Laurenzi M, Redfern KJ, Brune K. Comparison of rofecoxib, celecoxib, and naproxen on renal function in elderly subjects receiving a normal-salt diet. Clin Pharmacol Ther 2002; 72: 50-61.

12 Swan SK, Rudy DW, Lasseter KC,Ryan CF, Buechel KL, Lambrecht LJ, Pinto MB, Dilzer SC, Obrda O, Sundblad KJ, Gumbs CP, Ebel DL, Quan H, Larson PJ, Schwartz Jl, Musliner TA, Gertz BJ, Brater DC, Yao SL. Effect of cyclooxygenase-2 inhibition on renal function in elderly persons receiving a low-salt diet. Ann Intern Med 2000; 133 : $1-9$.

13 Schwartz Jl, Thach C, Lasseter KC, Miller J, Hreniuk D, Hilliard DA, Snyder KM, Gertz BJ, Gottesdiener KM. Effects of etoricoxib and comparator nonsteroidal anti-inflammatory drugs on urinary sodium excretion, blood pressure, and other renal function indicators in elderly subjects consuming a controlled sodium diet. J Clin Pharmacol 2007; 47: 1521-31. 
141983 Metropolitan height and weight tables. Stat Bull Metrop Life Found 1983; 64: 3-9. PMID: 6623350.

15 Schwartz Jl, De Smet M, Larson PJ, Verbesselt R, Ebel DL, Lins R, Lens S, Porras AG, Gertz BJ. Effect of rofecoxib on the pharmacokinetics of digoxin in healthy volunteers. J Clin Pharmacol 2001; 41: 107-12.

16 Snel S, Jansen JA, Pedersen PC, Jonkman JHG, van Heiningen PNM. Tiagabine, a novel antiepileptic agent: lack of pharmacokinetic interaction with digoxin. Eur J Clin Pharmacol 1998; 54: 355-7.

17 Harder S, Thürmann PA. Pharmacokinetic and pharmacodynamic interaction trial after repeated oral doses of imidapril and digoxin in healthy volunteers. Br J Clin Pharmacol 1997; 43:475-80.

18 Awni WM, Hussein Z, Cavanaugh JH, Granneman GR, Dube LM. Assessment of the pharmacokinetic interaction between zileuton and digoxin in humans. Clin Pharmacokinet 1995; 29 (Suppl. 2): 92-7.

19 Rodin S, Johnson B. Pharmacokinetic interactions with digoxin. Clin Pharmacokinet 1988; 15: 227-44.

20 Schwartz Jl, De Smet M, Larson PJ, Verbesselt R, Ebel DL, Lins R, Lens S, Porras AG, Gertz BJ. Effect of rofecoxib on the pharmacokinetics of digoxin in healthy volunteers. J Clin Pharmacol 2001; 41: 107-12.

21 Tanigawara Y. Role of P-glycoprotein in drug disposition. Ther Drug Monit 2000; 22: 137-40.
22 Reuning RH, Geraets DR. Digoxin. In: Applied Pharmacokinetics, 2nd edn, eds Evans WE, Schentag JJ, Jusko WJ. Spokane, WA: Applied Therapeutics, 1986; 570-623.

23 Williamson KM, Thrasher KA, Fulton KB, LaPointe NM, Dunham GD, Cooper AA, Barrett PS, Patterson JH. Digoxin toxicity: an evaluation in current clinical practice. Arch Intern Med 1998; 158: 2444-9.

24 Chapple DJ, Hughes R, Johnson BF. The relationship between cardiotoxicity and plasma digoxin concentration in conscious dogs. Br J Pharmacol 1976; 57: 23-7.

25 Cannon CP, Curtis SP, Bolognese JA, Laine L. Clinical trial design and patient demographics of the Multinational Etoricoxib and Diclofenac Arthritis Long-term (MEDAL) Study Program: cardiovascular outcomes with etoricoxib vs. diclofenac in patients with osteoarthritis and rheumatoid arthritis. Am Heart J 2006; 152: 237-45.

26 Cannon CP, Curtis SP, FitzGerald GA, Krum H, Kaur A, Bolognese JA, Reicin AS, Bombardier C, Weinblatt ME, van der Heijde D, Erdmann E, Laine L; MEDAL Steering Committee. Cardiovascular outcomes with etoricoxib and diclofenac in patients with osteoarthritis and rheumatoid arthritis in the Multinational Etoricoxib and Diclofenac Arthritis Long-term (MEDAL) programme: a randomised comparison. Lancet 2006; 368: 1771-81. 